\title{
Flexible High Capacitance Nanocomposite Gate Insulator for Printed Organic Field Effect Transistors
}

\author{
Amjad Rasul, ${ }^{1,2}$ Jie Zhang, ${ }^{1}$ Dan Gamota, ${ }^{1}$ Manish Singh,${ }^{2}$ Christos Takoudis ${ }^{2,3}$ \\ (1) Motorola Inc \\ (2) Department of Chemical Engineering \\ Physical Realization Research Center \\ (3) Department of Bioengineering \\ 1301 E. Algonquin Road \\ University of Illinois at Chicago \\ Schaumburg, IL 60196 USA \\ 851 S. Morgan Street \\ Chicago, IL 60607 USA
}

\begin{abstract}
Ceramic-polymer nanocomposite dielectric consisting of an epoxy solution with Propylene Glycol Methyl Ether Acetate as the solvent and Barium Titanate nanoparticles with capacitance in excess of $60 \mathrm{pF} / \mathrm{mm}^{2}$ was developed and utilized as the gate insulator for organic field effect transistors (OFETs). The high relative permittivity $(\kappa=35)$, bimodal nanocomposite utilized had two different filler particle sizes $200 \mathrm{~nm}$ and $1000 \mathrm{~nm}$ diameter particles. Bottom gate organic filed effect transistors were demonstrated using a commercially available printing technology for material deposition. A metal coated plastic film was used as the flexible gate substrate. Solution processable, p-type arylamine based amorphous organic semiconductor was utilized as the active layer. Fabricated OFETs with the solution processed nanocomposite dielectric had a high fieldinduced current and a low threshold voltage; these results suggest that the low operating voltage was due to the high capacitance gate insulator. In this paper, we review the characteristics of the nanocomposite dielectric material and discuss the processing and performance of the printed organic devices.
\end{abstract}




\section{Introduction}

Organic circuits with a performance suitable for certain applications have been demonstrated during the past few years. These applications include flat-panel displays, low-end smart cards, electronic identification tags as well as sensing devices [1-5]. The driving force for developing organic electronics is the fact that they are flexible, lightweight, and the prospect of low cost manufacturing. Due to the fact that polymers cannot withstand high processing temperature, one has to use low processing temperature techniques. The low processing temperature feature of the integrated circuits based on organic field effect transistors (OFETs) provides the opportunity for low cost processing [6].

Despite the significant progress in optimizing the performance of OFETs, the reported operational voltage of these devices is often too high causing their integration in low cost, disposable electronic products to be rather difficult. In order to be implemented as a viable technology, OFETs must offer a substantial performance advantage compared with the current technology. From the performance point of view, the most important parameters are charge carrier mobility, on/off current ratio and the operational voltage range. Up to now, the highest mobility values, superior to amorphous silicon (a:Si), have been obtained for pentacene-based OFETs with sufficiently high on/off ratios [7]. However, the operating voltages required to produce such performances were un-practically high, around $100 \mathrm{~V}$. The reason for that was shown to be the mobility dependence on the accumulated charge in the OFET channel. Since this charge is proportional to both the dielectric constant and the gate voltage, it has been suggested that the use of high dielectric constant materials will allow the necessary charge to accumulate at much lower voltages [8]. High gate capacitance is a desirable parameter for OFETs, because it allows a higher charge density to be induced at lower voltages and it increases drive capability. 
The capacitance can be increased by using a thinner dielectric or by using a high permittivity insulator material. High permittivity inorganic materials such as barium titanate and barium strontium titanate were used to reduce operating and threshold voltages [9]. Increased capacitance means that the semiconductor may operate in a different regime, with significantly higher density of carriers in the channel and/or reduced field acting perpendicular to the direction of transport. Increasing the gate capacitance enables trap states responsible for a threshold voltage to be filled at a much lower gate voltage, and further carrier accumulation will therefore result in free carrier flow.

We report the use of solution processed high $\kappa$ barium titanate $\left(\mathrm{BaTiO}_{3}\right.$, denoted as $\mathrm{BTO}$ from now on) nanocomposite as dielectric material for low operating voltage printed OFETs. Polymeric OFET insulators (e.g. Poly (methylmethacrylate) [10]. Benzocyclobutene [11], Poly4-vinylphenol [12], and Polyvinyl alcohol [13], etc), which are solution processable, have to be fairly thick to avoid pin-holes and their dielectric constants $k$ are rather low. These gate insulators produce low gate capacitance and consequently, high device operational voltage exceeding 30 V. Metal oxides ( $\mathrm{HfO}_{2}$ [14], $\mathrm{Gd}_{2} \mathrm{O}_{3}$ [15], $\mathrm{Al}_{2} \mathrm{O}_{3}$ [16], $\mathrm{Ta}_{2} \mathrm{O}_{5}$ [17], etc.), have a higher dielectric constant than polymers, but their sputtering process is rather expensive which significantly increases the device's manufacturing cost. Additionally, the high processing temperature required for metal oxides is not compatible with the flexible polymeric substrates used in most printed electronics applications. $\mathrm{SiO}_{2}$ has often been used as an OFET insulator, but more for the reason of availability rather than performance, as $\mathrm{SiO}_{2}$ does not display much higher capacitance than polymers $(\kappa \sim 3.9)$. Self assembly technology was used to deposit top gate dielectric layer formed with $\mathrm{SiO}_{2}$ nanoparticles [18]. Self assembly was also utilized to modify an $\mathrm{SiO}_{2}$ thin layer for use in an OFET that used evaporated Pentacene organic 
semiconductor [19]. Recently, high $\kappa$ titanium dioxide nanoparticles [20] have been used to formulate nanocomposite gate insulators with higher field-induced current than that of conventional devices due the increased insulator dielectric constant. Titanium dioxide has been used as an inorganic oxide core of a polymer shell nanocomposite high $\kappa$ dielectric for OFETs [21].

In this work, bottom gate OFETs were fabricated on a flexible metal coated plastic substrate with the BTO/epoxy nanocomposite as the gate insulator. As active material we have used a solution processed polytriarylamine (PTAA) based_organic semiconducting material that was spray coated over the metal electrodes and the gate insulator. BTO nanocomposite dielectric properties were investigated. High relative permittivity $(\kappa=35)$ was obtained with the $60 \mathrm{v} \%$ bimodal BTO nanoparticle loading in the dry film. The fabricated OFETs with the nanocomposite dielectric layer had a high field-induced current with a low threshold voltage.

\section{Experimental Details}

Bottom gate OFETs were fabricated using a metal coated plastic film as the substrate and gate electrode as shown in Figure 1. A specialty bimodal nanocomposite was formulated by Huntsman Advanced Materials for our evaluation. A two-part epoxy solution (Probelec ${ }^{\circledR}$ 81, Huntsman, Switzerland) with propylene glycol methyl ether acetate as the solvent was utilized as the polymer matrix [22]. To insure homogeneous dispersion of the BTO particles in the polymer matrix, a non-ionic fluorosurfactant of the polyalky ether type 116 (CHEMGURAD S-222N) was used as BTO particle surfactant. The curing agent used in this study was an anhydride crosslinking agent (1-Methoxy-2-Propanol, LMB 7228) form Huntsman Advanced Materials.[22] The barium titanate ceramic filler consisted of two different particle sizes: $200 \mathrm{~nm}$ and $1000 \mathrm{~nm}$ 
diameter at a particle distribution ratio of $1: 3$ by weight [22]. In bulk BTO ceramics, grain size has a strong effect on the dielectric constant at room temperature for the grain size below approximately $10 \mu \mathrm{m}$ [23]. Dielectric constant increases with decreasing grain sizes up to a maximum at approximately $1000 \mathrm{~nm}$. Below this size, dielectric constant sharply decreases again, in conjunction with the reduction of tertragonality and of remanent polarization. Crystal structure of BTO powder changes with its size. According to Uchino [24], at room temperature, tetragonality decreases as BTO powder size decreases slowly down to about $300 \mathrm{~nm}$. Below this size it sharply decreases again, and at approximately $100 \mathrm{~nm}$ it disappears, which means cubic or pseudocubic structure with very low dielectric constant. Having the selected two different particle sizes (200 and $1000 \mathrm{~nm}$ ) enhances the BTO particle surface coverage in the cured film and results in a higher nanocomposite dielectric constant. Previous studies by Cho [25] had indicated that a higher dielectric constant is achieved with a bimodal nanocomposite compared to a unimodal one with the same particle loading. Our dielectric characterization results agreed with this finding. We formulated unimodal $(200 \mathrm{~nm}$ BTO particles only and $1000 \mathrm{~nm}$ BTO particles only) and bimodal nanocomposites (200 and $1000 \mathrm{~nm}$ BTO particles) at the same loading level of $60 \mathrm{v} \%$. The BTO/Epoxy nanocomposite gate dielectric was roller coated at a rate of 30 inches per minute using a $1 / 2$ inch diameter stainless steel rod. Cured nanocomposite dielectric film thickness was about $9 \mu \mathrm{m}$ as determined using an optical profilometer (WYKO Systems) and $5 \mu \mathrm{m}$ thick pad printed metal electrodes were used for both the sources and drain. The channel width and length were $1000 \mu \mathrm{m}$ and $150 \mu \mathrm{m}$, respectively. Approximately $200 \mathrm{~nm}$ thick PTAA amorphous organic semiconductor was spray coated on top of the source and drain. PTAA polymers are p-type materials, only hole transient is observed. The bulk mobility of the 
PTAA polymer is approximately $10^{-2} \mathrm{~cm}^{2} / \mathrm{V}$.s The OFET fabrication process was done under ambient condition.

Nanocomposite dielectric cure profile was determined using Differential Scanning Calorimeter (DSC) model 2200 from TA Instruments. The surface morphology of the BTO/Epoxy nanocomposite was examined using both optical microscopy as well as Scanning Electron Microscopy (SEM). To determine the dielectric properties of the nanocomposite film, parallel plate capacitors were fabricated using the same substrate and electrode metals. The fabricated capacitors had a $5 \mu \mathrm{m}$ dielectric thickness as measured using an optical profilometer. Capacitance measurements were done using an HP 8753C Network Analyzer from $100 \mathrm{~Hz}$ to $100 \mathrm{KHz}$. The electrical characterization of the printed OFETs was done using a Keithly 2400 Source meter while devices were held under vacuum in a dark chamber.

\section{Results and Discussion}

Figure 2 shows the DSC results of $60 \mathrm{v} \%$ filler BTO/Epoxy nanocomposite. After curing, the glass transition temperature ( $\mathrm{Tg}$ ) was measured using DSC under modulated mode. The cure reaction stars at about $65^{\circ} \mathrm{C}$ and the curing peak appeared at approximately $85^{\circ} \mathrm{C}$. The Tg. of the nanocomposite is around $70^{\circ} \mathrm{C}$. To ensure complete curing, the nanocomposite was cured at $150{ }^{\circ} \mathrm{C}$ for 1 hour in a ventilated oven. The curing behavior displayed makes the nanocomposite suitable as a printed insulator on flexible organic substrates.

It must be noted, however, that the permittivity is not the only parameter for the optimal material choice, as the dielectric is also required to have high dielectric breakdown, yield a uniform and defect- and pinhole-free film, and be deposited from an orthogonal solvent to the semiconductor. Analysis of the morphology of the nanocomposite of layers is therefore important for overall 
performance, and this can be achieved using several methods such as SEM and optical surface analysis. BTO particle surface coverage was quite uniform as can be seen from the SEM image of the cured dielectric shown in Figure 3. The dielectric curing process is partially responsible for the minimal particle agglomeration seen in the SEM image. Having two different particle sizes enhances the BTO particle surface coverage in the cured film and results in a higher nanocomposite dielectric constant. A significant advantage of the bimodal nanocomposite is the considerable minimization of nano-particle agglomeration as compared to a unimodal nanocomposite. Significant particle agglomeration was detected when unimodal nanocomposites of $200 \mathrm{~nm} \mathrm{BTO}$ and $1000 \mathrm{~nm}$ BTO particles were used which resulted in a very high surface roughness. This high surface roughness made it prohibitive to process thin subsequent layers. Optical inspection of the surface of bimodal and unimodal nanocomposite surfaces revealed a significantly smoother bimodal nanocomposite surface. The BTO/Epoxy nanocomposite dielectric had a breakdown voltage of $23 \mathrm{KV} / \mathrm{mm}$ at $1000 \mathrm{~Hz}$.

Dielectric characterization of the nanocomposite dielectric was done through the fabrication of parallel plate capacitors. A Trans Tech SEALCUP 60, all-mechanical, cam-operated bench model pad printing machine with a hermetically sealed ink cup was used to print the BTO nanocomposite dielectric as well as the top silver electrode layer. A metal coated plastic film was used as the flexible bottom electrode substrate. The fabricated capacitors had a $5 \mu \mathrm{m}$ dielectric thickness as measured using an optical profilometer. Capacitance measurement was done using an HP 8753C Network Analyzer from $100 \mathrm{~Hz}$ to $100 \mathrm{KHz}$, and is shown in Figure 4. Bimodal nanocomposite capacitance density was determined to be $62 \mathrm{pF} / \mathrm{mm}^{2}$ with a dielectric loss tangent $(\tan \delta)$ of only $3 \%$ at $30 \mathrm{KHz}$. Dielectric constant value was calculated from the capacitance $\mathrm{C}$ data using the equation: 


$$
\mathrm{C}=\varepsilon_{0} \varepsilon_{\mathrm{r}} \mathrm{A} / \mathrm{t}
$$

where, $\varepsilon_{0}$ is dielectric constant of the free space $\left(8.85 \times 10^{-12} \mathrm{~F} / \mathrm{m}\right), A$ is the area of the electrical conductor plate, $\mathrm{t}$ is the thickness of the insulating layer, and $\varepsilon_{\mathrm{r}}$ is the dielectric constant of the insulator layer. The nanocomposite dielectric constant (к) was calculated to be 35 .

Optical profiling (white light interferometry) using a WYKO NT8000 system (Veeco Instruments Inc) was used to determine the nanocomposite thickness and surface roughness. Optical profiling is a standard technique for accurately assessing step heights and other surface topography. Utilizing the Enhanced Vertical Scanning Interferometry mode of the WYKO NT8000 optical profilometer, the printed nanocomposite dielectric thickness in the OFET devices was about $9 \mu \mathrm{m}$ as seen in the 2-D Plot in Figure 5. A comparison of the dielectric properties of the bimodal nanocomposite with the unimodal nanocomposite is illustrated using the capacitance frequency data shown in Figure 6. The bimodal nanocomposite displays higher capacitance than both unimodal nanocomposites at the same particle loading of $60 \mathrm{v} \%$._ This is a combined result of the highest dielectric constant at $\sim 1000 \mathrm{~nm}$, higher packing with the bimodal nanocomposite, high surface to volume ratio, and of significant tertragonality and remanent polarization. $[22,24]$

Printed OFETs were electrically characterized using a Keithly 2400 source meter while the devices were held under vacuum in a dark chamber. Figure 7 shows the drain-source current $\left(I_{D}\right)$ verses gate-source voltage $\left(\mathrm{V}_{\mathrm{G}}\right)$ of printed OFETs with a $\mathrm{BTO} /$ Epoxy nanocomposite gate insulator at a drain-source voltage $\left(\mathrm{V}_{\mathrm{D}}\right)$ of $-40 \mathrm{~V}$. The device transfer characteristics shown are for a device that had a channel width (W) of $1000 \mu \mathrm{m}$ and a channel length (L) of $150 \mu \mathrm{m}$. 
The high current output obtained is due in part to the high capacitance nanocomposite gate insulator. Further reducing the insulator thickness can lead to even higher current values. The mobility was extracted from the slope of $\left(\mathrm{I}_{\mathrm{D}}\right)^{1 / 2}$ verses $\left(\mathrm{V}_{\mathrm{G}}\right)($ Fig. 8$)$ in the saturated region of the transfer curve where the source-drain current $\left(I_{D}\right)$ is independent of the source-drain voltage $\left(V_{D}\right)$ and approximately follows the equation:

$$
\left|\mathrm{I}_{\mathrm{D}}\right|=\mathrm{W} \mathrm{C} \mu\left(\mathrm{V}_{\mathrm{G}}-\mathrm{V}_{\mathrm{th}}\right)^{2} /(2 \mathrm{~L})
$$

where $\mathrm{C}$ is the capacitance per unit area of the nanocomposite insulator, $\mathrm{W}$ is the channel width, $\mathrm{L}$ is the channel length, $\mathrm{V}_{\text {th }}$ is the threshold voltage, and $\mu$ is the field-effect mobility. The key to low-voltage operation is the reduction of the threshold voltage and the subthreshold slope. The field-effect charge mobility was a $1.2 \times 10^{-4} \mathrm{~cm}^{2} / \mathrm{V}$.s and the on/off ratio was in the range of $10^{3}$. The threshold voltage was only $4 \mathrm{~V}$. The on/off ratio and threshold voltage represent significant improvement over data reported by Zielke et al.[26]. We believe the use of a bimodal nanocomposite that has lower surface roughness and higher capacitance compared to a unimodal nanocomposite play a major role in achieving improved performance. Note that the maximum mobility of the used PTAA semiconductor is $3 \times 10^{-3} \mathrm{~cm}^{2} / \mathrm{V}$.s in an optimized device.

\section{Conclusions}

In summary, we have demonstrated the potential of using solution processed, high capacitance BTO/Epoxy nanocomposite as an insulator for printed organic electronics by preparing a highperformance device with low-voltage operation. We have also shown that the BTO/Epoxy nanocomposite dielectric can be used to obtain organic transistors with good electrical properties with an economic and easy process. The device we fabricated with the nanocomposite insulator was found to have a field-effect mobility of $1.2 \times 10^{-4} \mathrm{~cm}^{2} / \mathrm{V}$.s and a threshold voltage of only 
$4 \mathrm{~V}$. This combination of favorable properties demonstrates that OFETs can be operated at low operating voltages.

\section{Acknowledgments}

We are grateful to the Motorola Physical Realization Research CoE (PRRC) staff for their

contributions. We are also grateful to Huntsman Advanced Materials for their help with formulating the nanocomposite material. 


\section{References}

[1]. C. Dimitrakopoulos, and D. Mascaro, IBM J. Res. and Dev. 45 (2001) 11.

[2[. H. Edzer, A. Huitema, G. Gelinck, J. Bas, P. Van der Putten, K. Kuijk, K. Hart, E. Cantatore, D. de Leeuw, Adv. Mater.14 (2002) 1201.

[3]. B. Crone, A. Dodabalapur, R. Sarpsskar, A. Gelperin, H. Katz, Z. Bao, J. Appl. Phys. 91 (2002) 10140.

[4]. T. Jackson, Y. Lin, D. Gundlach, and H. Klauk, J. Sel. Topics Quant. Elec. 4 (1998) 100.

[5]. G. Gelinck, T Genus, D. De Leeuw, Appl. Phys. Lett. 77 (2000) 1487.

[6]. F. Garnier, R. Hajlaoui, M.E. Kassmi, Appl. Phys. Lett. 73 (1998) 1721.

[7]. S. Nelson, Y.Y. Lin, D.J. Gundlach, T.N. Jackson, Appl. Phys. Lett. 72 (1998) 1854.

[8]. C.D. Dimitrakopoulos, S. Purushothaman, J. Kymissis, A. Callegari, J.M. Shaw, Science 283 (1999) 822.

[9]. C. Dimitrakopoulos, K. Kymissis, S. Purushothaman, D. Neumayer, P. Duncombe, R. Laibowitz, Adv. Mater.11 (1999) 1372.

[10]. S. Uemura, M. Yoshida, S. Hoshino, T. Kodzasa, T. Kamata, Thin Solid Films 438-439 (2003) 378 .

[11]. L.L. Chua, P.K.H. Ho, H. Sirringhaus, R.H. Friend, Appl. Phys. Lett. 84 (2004) 3400.

[12]. H. Klauk, M. Halik, U. Zschieschang, G. Schmid, W. Radlik, W. Weber, Appl. Phys. Lett. $92(2002) 5259$.

[13]. R. Parashkov, E. Becker, G. Giniev, T. Riedl, H.H. Johannes, W. Kowalsky, J. Appl. Phys. 95 (2004) 1594. 
[14]. M.R. Yisokay, J.J. Chambers, A.L.P. Rotondaro, A. Shanware, L. Colombo, Appl. Phys. Lett. 80 (2002) 3183.

[15]. S.J. Kang, K.B. Chung, D.S. Park, H.J. Kim, Y.K. Choi, M.H. Jang, M. Noh, C.N. Whang, Synth. Met.146 (2004) 351.

[16]. W. Kalb, P. Lang, M. Mottaghi, H. Aubin, G. Horowitz, M. Muttig, Synth. Met.146 (2004) 279.

[17]. J. Yuan, J. Zhang, J. Wang, D. Yan, W. Xu, Thin Solid Films 450 (2004) 316.

[18]. T. Cui, G. Liang, Appl. Phys. Lett. 86 (2005) 86.

[19]. L. A. Majewski, M. Grell, Synth. Met. 151 (2005) 175.

[20]. F. Chen, C. Chun, J. He, Y. Yang, J. Lin, Appl. Phys. Lett. 85 (2004) 3295.

[21]. A. Maliakal, H. Katz, P. Cott, S. Subramoney, P. Mirau, J. Am. Chem. Soc. 127 (2005) 14655.

[22] A. S. Rasul, Ph.D. Dissertation, Department of Chemical Engineering, University of Illinois at Chicago, USA, 2007.

[23]. G. Arlt, D. Henning, G. de With, J. Appl. Phys. 58 (1985) 1619.

[24]. K. Uchino, E. Sadanaga, T. Hirose, J. Am. Ceram. Soc. 72 (1989) 1555.

[25]. S. Cho, J. Lee, J. Hyun, K. Paik, Mater. Sci. Eng. B 110 (2004) 233.

[26]. D. Zielke, A. Hubler, U. Hahn, N. Brandt, M. Bartzsch, U. Fugmann, T. Fischer, J. Veres, S. Ogier, Appl. Phys. Lett. 87 (2005) 87. 


\section{List of Figures}

Figure 1. Schematic cross section of a bottom gate organic filed effect transistor with a BTO nanocomposite gate insulator.

Figure 2. Curing profile of the BTO/Epoxy nanocomposite.

Figure 3. SEM micrograph of BTO nanoparticles top surface in the cured dielectric.

Figure 4. BTO/Epoxy nanocomposite dielectric capacitance $(\mathrm{nF})$ as a function of frequency (KHz) (5 $\mu \mathrm{m}$ nanocomposite thickness).

Figure 5. Top surface optical scanning of nanocomposite dielectric coated on aluminum. (nanocomposite dielectric thickness : $9 \mu \mathrm{m}$, average surface roughness: $675 \mathrm{~nm}$ )

Figure 6. BTO/Epoxy Bimodal and Unimodal Nanocompsosite capacitance vs. frequency data for the same thickness capacitors. All three nanocomposites had 60v\% BTO loading with $7 \mu \mathrm{m}$ thickness.

Figure 7. Transfer characteristics (drain current ID vs. gate voltage $\mathrm{V}_{\mathrm{G}}$ ) for OFET with $12 \mu \mathrm{m}$. thick bimodal nanocomposite gate insulator, at constant drain voltage $\mathrm{V}_{\mathrm{D}}=-40 \mathrm{~V}$.

Figure 8. Drain current (ID) vs. gate voltage $(V g)$ and square root of drain current $\left(I_{\mathrm{D}}\right)^{1 / 2}$ vs. gate voltage $(V g)$ characteristics of PTAA OFET with BTO/Epoxy nanocomposite gate insulator (Channel L/W: $150 \mu \mathrm{m} / 1000 \mu \mathrm{m}$, threshold voltage: $4 \mathrm{~V}, V_{\mathrm{D}}=-40 \mathrm{~V}$ ) 